\title{
Phenomena of Marcus Gunn (MGJWS) or The Jaw winking Syndrome
}

\author{
Sharanya Nath ${ }^{1}$ \\ ${ }^{1}$ Affiliation not available
}

August 3, 2021

\begin{abstract}
In individuals showing Marcus Gunn phenomena, it is characterized when the upper eyelid shows involuntary movement (continuous rising motion - more like a wink) every time the jaw is moved, like while eating. It is a congenital disease as it is generally seen to be present since birth, and in certain cases various other anomalies and abnormalities in the eye may also occur, for example: Strabismus and other vision impairments. Despite the fact that it for the most part influences one eye, it can influence the two eyes in uncommon cases.
\end{abstract}

\section{Study of Marcus Gunn Phenomena}

Marcus Gunn phenomena also known as Jaw winking condition can be observed in congenital blepharoptosis. This phenomena was first depicted by a Scottish ophthalmologist, named Dr.Robert Marcus Gunn in the year 1883. This disorder for the first time was observed in a 15-year-old young lady as one-sided ptosis related with the upper eyelid constriction on a similar side.

Marcus Gunn jaw-winking syndrome (MGJWS) over the years have also acquired various names such as, Pterygoid-levator synkinesis, Marcus Gunn jaw winking trigemino-oculomotor synkinesis, Marcus Gunn ptosis, and Maxillopalpebral synkinesis. Synkinesis or synkinetic movement is a neurological condition wherein an intentional movement of the muscle causes the synchronous constrictions and contraction of other involuntary muscles. In MGJWS, during masticating motions of the jaw, it is linked to synkinetic movements of the upper eyelid. This usually is unilateral, but can also be bilateral in some cases. This is caused by a persistent connection which is abnormal linking the superior division of oculomotor nerve with the motor branches of the trigeminal nerve.

\section{Causes and speculation}

Studies show that people with Marcus Gunn disease may not necessarily have any past familial records concerning this disorder. Hence it can be safely said that this disorder is not passed down within generations. However, some cases do report multiple cases of this phenomena occurring within one family at a given point of time, but so far the genetic lineage causing this phenomena is unknown.

Roughly fifty percent of the MGJWS cases are discovered to be congenital. Since any hereditary relationship linking to this disorder is yet to be discovered, it can be assumed that it occurs due to genetic variations during foetal development. It has been seen that there could be various reasons leading up to Marcus Gunn 
phenomenon and in certain cases children born with this disorder are also born with other congenital defects caused by genetic or chromosomal mutation.

\section{Inheritance}

In every living being, the nucleus of every cell contains the genetic material which is passed down within species and is the unique identity of an individual. The humans, contain chromosomes in the nucleus which are 46 in number. These are further paired in 23 groups, numbered from 1 to 22 and known as Autosomal chromosomes. The $23^{\text {rd }}$ pair known as the sex chromosome has 2 designated chromosomes namely, $\mathrm{X}$ and $\mathrm{Y}$. Apart from the 22 autosomal pair, males have XY sex chromosome pair and females have XX. On the basis of banding patterns, chromosomes are then subdivided and each band is numbered. These bands contains thousands of genes whose location are distinguished by the numbering. Furthermore, every chromosome consist of a long arm ' $q$ ' and a short arm 'p'. For example, $18 \mathrm{q} 12$ refers to the $12^{\text {th }}$ band of the long arm in the $18^{\text {th }}$ chromosome.

Genomic disorders such as Marcus Gunn phenomena hence can be ascertained on the basis of gene combinations for a particular trait which are inherited from either of the parental chromosome. In the case of MGJWS, some clinical researchers hypothesize that there is a chance of around half of the MGJWS cases can be ascertained through genetic combinations. The exact location of the gene on the chromosomal sites and its nature is still unknown for this disorder, but some studies suggest that there is a chance that it could be disseminated as an autosomal dominant trait.

\section{Diagnosis and Therapy}

- In infants, the visual acuity is checked to see if there is any presence of Lazy eye or Amblyopia (a disorder in which an abnormal visual development leads to reduced vision capabilities in one of the eyes).

- Tests like Pupillary examinations to check focus of the eye and Cycloplegic refraction tests are carried out to find any disorders wherein the eye might have varying refractive powers (Anisometropia).

- Ptosis can be assessed by considering the degree of it (refer to the table below) that is, from mild ptosis to severe ptosis. The head position is evaluated, as in a child with ptosis lifting of the chin is seen to attain better vision. Vision loss may occur in cases wherein if ptosis remains untreated. At severe stage surgery can be considered.

\begin{tabular}{ll}
\hline Type & Degree \\
\hline Mild & Maximum up to 2 millimetres \\
Moderate & From 2 to 5 millimetres \\
Severe & More than 5 millimetres \\
\hline
\end{tabular}

- Conditions arising due to MGJWS such as vertical strabismus, double elevator palsy and horizontal strabismus can be controlled and cured up to a level using medications, eyeglasses and in critical cases surgery.

- Keeping the eyes of a child affected with Marcus Gunn phenomena in its primary gaze position MRD (Marginal reflex distance) can be measured.

\section{Type of MRD Measurement}

MRD $1 \quad$ From the corneal reflex to the margin of the central upper lid 


\begin{tabular}{ll}
\hline Type of MRD & Measurement \\
\hline MRD 2 & From the corneal reflex to the margin of the central lower lid \\
MRD 3 & From the corneal reflex to the margin of the central upper lid making the child take his eyes to extreme \\
\hline
\end{tabular}

\section{References}

1. Sundareswaran S, Nipun C A, Kumar V. Jaw - winking phenomenon: Report of a case with review of literature. Indian J Dent Res 2015;26:320-3

2. Shah AD, Kumar AB, Kothari K. Bilateral Marcus Gunn jaw winking synkinesis with monocular elevation deficiency: a case report and literature review. Int Ophthalmol. 2012 Apr;32(2):199-201

3. Marcus-Gunn jaw Winking Ptosis . EyeWiki. (2021, February 6). https://eyewiki.aao.org/MarcusGunn_Jaw_Winking_Ptosis.

4. Senthilkumar VA, Tripathy K. Marcus Gunn Jaw Winking Syndrome. [Updated 2021 Feb 14]. In: StatPearls [Internet]. Treasure Island (FL): StatPearls Publishing; 2021 Jan-. Available from: https://www.ncbi.nlm.nih.gov/books/NBK559058/

5. (Marcus Gunn Phenomenon| Genetic and Rare Diseases Information Center (GARD) - an NCATS Program, n.d.)

6. NORD - National Organization for Rare Disorders. (2015, February 11). Marcus Gunn Phenomenon . https://rarediseases.org/rare-diseases/marcus-gunn-phenomenon/ 